\title{
The infrared Temperature Measurement System using STM32
}

\author{
Wenbo Han ${ }^{1, a}$, Jianlin Song ${ }^{2, \text { b * }}$ \\ ${ }^{1}$ Changchun University of Science and Technology, China \\ ${ }^{2}$ Changchun University of Science and Technology, China \\ ahanwenbo@cust.edu.cn, b15143014944@163.com
}

keywords: Infrared Temperature Measurement; STM32; LabVIEW.

Abstract.Infrared temperature measurement technology is widely used in non-contact temperature measurement field. The designed system adopts infrared measuring temperature sensor of TN series to measure the target temperature and displays the data on the LCD screen with the STM32. At the same time, it combines with the LabVIEW programming to achieve the purpose of the remote control. Different from the non-contact temperature measurement technology, this system holds more advantages, which response fast, measure widely and so on. Meanwhile, convenience is also been provided for the daily life and industrial production.

\section{Introduction}

It's a traditional way to measure temperature with the contact method. However, it can only measure an object which reaches thermal equilibrium state with temperature sensor. The direct result is a long response time, easily affected by environmental temperature and declined accuracy of measurement. Compared with the traditional measuring way, infrared temperature measurement technology own more advantages at the accuracy of measurement. Meanwhile, it is convenient of detecting the temperature with a fast speed. Because of that, the infrared temperature measurement technology becomes one of the most important detecting methods. This paper will introduce a device using the infrared temperature measurement technology. The device uses TN901 as the sensor to measure the temperature. It uses STM32 as the master and uses TN901 and DS1624 as the slaves. At the same time, the STM32 can transfer the actual temperature to the principle computer through the network. The principle computer uses LabVIEW as the software to control the communication with the STM32. This device can measure the temperature any time any where. It brings great convenience for long-distance observation target temperature and operating the machine system.

\section{The Principle}

Infrared radiation is one of the most widely existing in the nature of the electromagnetic wave radiation, which is based on any object in a conventional environment will produce their own molecular and atomic motion without rules. Meanwhile, the object will radiate energy ceaselessly. Molecules and atoms move more intense, the greater the radiation energy. On the contrary, the radiation energy will be less. Therefore, we can measure the infrared energy so as to detect the temperature of the object. That's the objective basis of infrared radiation temperature measurement.

According to the Stephen Boltzmann law, the higher the temperature of the object, the more energy will be out of its radiation. When the temperature is $\mathrm{T}$, the intensity of the object radiated in all wavelengths is: 


$$
\mathrm{M}_{\mathrm{b}}=\varepsilon \sigma \mathrm{T}^{4}
$$

In the Equation, the $\sigma$ is the Stephen Boltzmann constant; the $\mathrm{T}$ is the absolute temperature of the objects; the $\varepsilon$ is the normal emissivity of the objects surface, if the object is an absolute black, the $\varepsilon$ is one, or else it is less than one but larger than zero.

The infrared measuring sensor receives the energy which is radiated by the target, then the sensor transfer the energy into electrical signal. The signal will be magnified by the amplifier and changed to the temperature of the object to be measured.

TN901, the infrared sensor, is a good example. Its principle diagram is shown in Fig. 1. The infrared pyroelectric sensor is located at the focus of a concave mirror which is after a filter. So the sensor can receive the infrared energy with concentration. After this, an amplifier is designed to magnify the signal. Then an analogy-digital converter converts the analog signal to digital signal. Finally, according to the related formula of Stephen Boltzmann law and Wayne displacement law, we can calculate the surface temperature of the object to be tested.

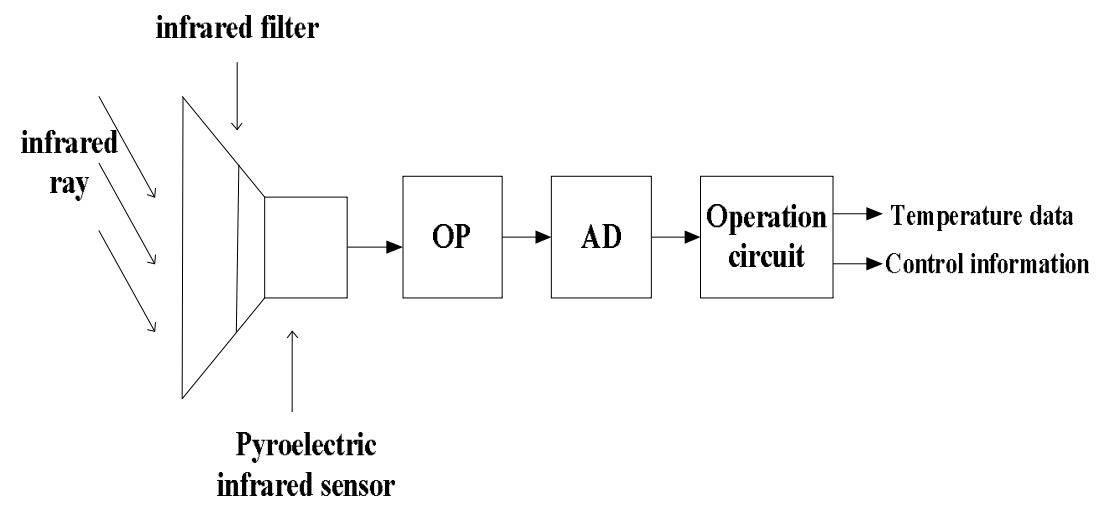

Fig. 1 the principle diagram of TN901

\section{Design Scheme}

The infrared temperature measurement system is composed of several big modules, respectively is the STM32 master control module, the infrared temperature measurement module, the display module, the PC remote control module and the power module. The system overall frame is shown in Fig. 2. The STM32 control the TN901 to temperature acquisition, then the TN901 exchange data with STM32. The STM32 will display the real time temperature on the LCD screen. As long as under the condition of network allows, the STM32 can realize the communication with the LabVIEW at any time. The STM32 use the protocol of TCP/IP to transfer data to PC, the PC use the software LabVIEW to control the temperature acquisition start or stop in a remote mode. This function will give the user convenience at any time checking the situation of the target temperature and operating the measuring system.

In order to present the advantages of fast response, measurement precision of the infrared technology, we use the contacted measuring unit DS1624 to collected temperature. By contrast, the infrared measuring technology will be very good highlight the advantages. 


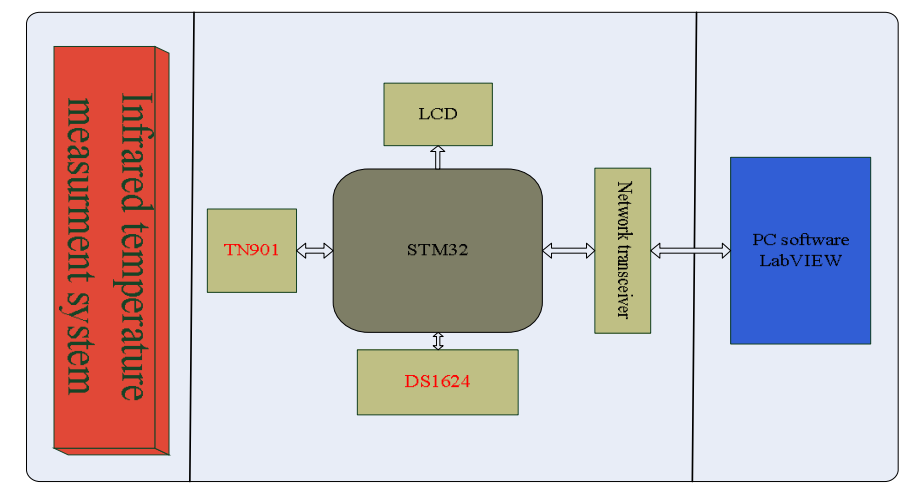

Fig.2 The overall Frame

\section{Program Design}

This system mainly includes two parts of program design, respectively is the process of the lower machine and the PC program design. The lower machine program design mainly includes the capturing temperature and displaying. The PC program design mainly includes the remote monitoring of the target temperature captured by the lower machine and the state of the lower machine.

\section{Hardware Design Program}

According to the requirements of structured programming, we use modularization and structured programming mode to design the program. Based on the function of the system, the program module contains the following: the main program module, power control module, temperature measurement module, LCD module, interrupt module, the DMA function module and so on. The detailed program schedule is shown in Fig. 3.

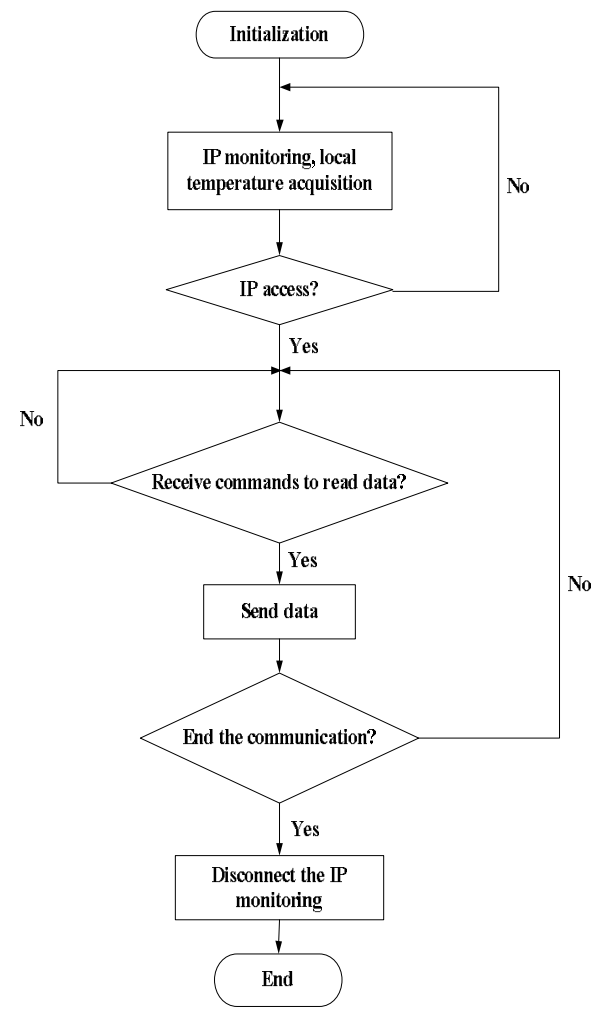

Fig.3 Hardware Program Flow Chart 
The main Program Module

The main program module mainly complete system initialization and TCP/IP status listening. The system initialization includes collecting temperature initialization and the LCD initialization. The TCP/IP status listening includes opening the TCP/IP network, querying a PC access or not. If there is a PC access, the STM32 will enter a DMA interrupt and wait for the PC transfer the command of starting to collect the temperature. The STM32 will transfer the temperature which collected by the TN901 to the PC.

Temperature Measurement Module

Temperature measurement module mainly include non-contact infrared temperature measurement module and contact temperature measurement module, in the process of testing, we use a heat source to provide a relatively stable real environment. The infrared temperature measurement module TN901 collect environment and target temperature and send the data to PC by bus. The temperature is shown on the screen. The contacted temperature unit DS1624 is also used to collect the temperature of the target and then display it on the screen.

Display Module

The system adopts LCD display the temperature. The Fig. 4 shows the real time temperature of the target. The blue curve represents the temperature which TN901 collected. The green curve represents the temperature which DS1624 collected. Through this figure, we can clearly find that the infrared measuring temperature sensor TN901 response more quickly. It's really the advantage of the sensor.

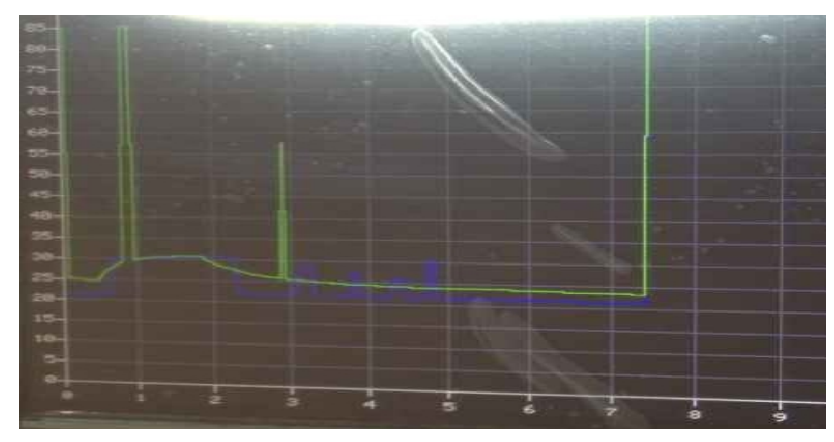

Fig.4 Temperature on the LCD screen

Software Program Design

The design of the system software mainly completed the project that read the temperature collected by the sensor and manipulate the lower machine. The Fig. 5 shows all the consequences of the program. At first, we use LabVIEW set the IP address and if the network is OK, the PC and the STM32 will establish communication. PC can send the command to start the temperature collecting or stop it. So we can control the STM32 at any time no matter where you stay. At the same time, it also saves the loss of the equipment. 


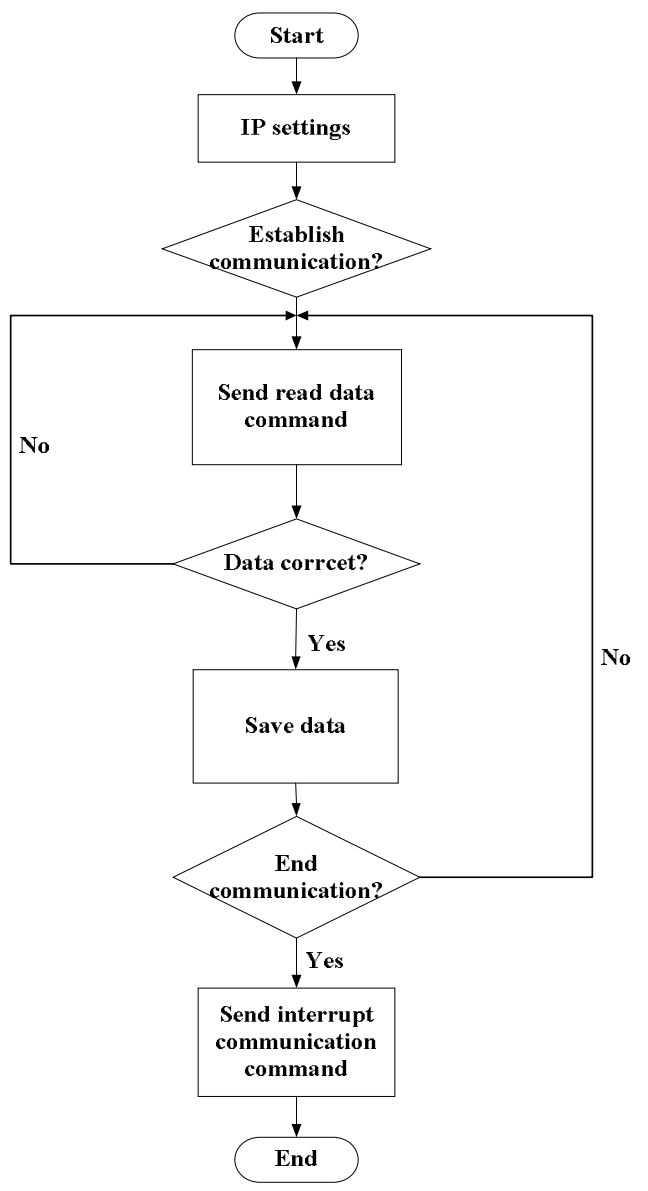

Fig.5 Software Program Flow Chart

The Fig. 6 shows the PC program. In the process of experiment, open the heat source to simulate the temperature of the environment change, the circle 1 means open the simulate heat source, and to set the temperature 28 degrees. Then wait for a few minutes, stop the heat source, the temperature then go down to the ambient temperature, the circle 2 shows the point. From the two different curve, we should find that the non-contact temperature measurement technology has clear advantage of fast response. It is convenient to put this device on difficult environment. And it will solve a series of problems.

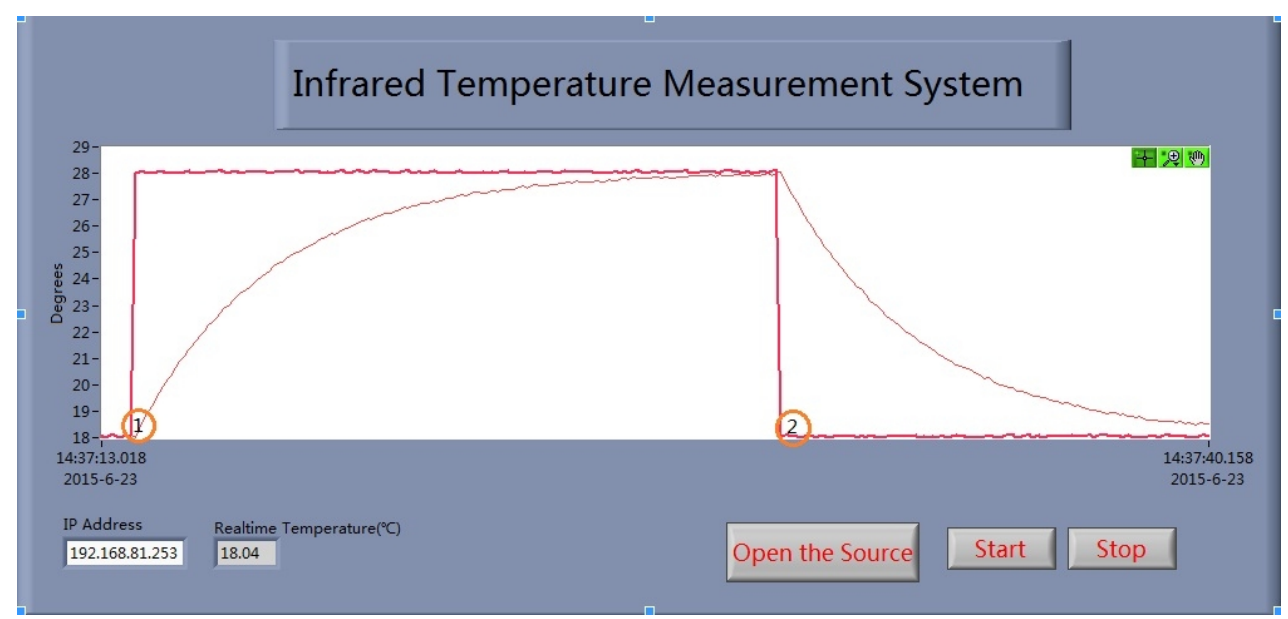

Fig.6 PC program 


\section{Conclusions}

This article introduces an infrared temperature measurement system based on STM32.This system realizes the function of the following points. First, it can get the accurate measurement of the target temperature and the environmental temperature. Second, display the temperature on the LCD screen. Third, remotely check the temperature and operate a machine to start or stop temperature measuring.

In the process of test, we use a heat source to simulate the different ambient. In this way, we know the non-contact measuring technology has more good advantages than contact measuring technology. At the same time, we use LabVIEW to realize the remote temperature observation. This can widely prolong the service life of the equipment.

\section{References}

[1] Haoqiang Tang. C program design(second edition) . Beijing: Tsinghua university press. (2003)

[2] Zhibo Chen, Chun-ling Wang. Objective programming language for $\mathrm{C}++$. Beijing: people's posts and telecommunication publishing house. (2002)

[3] Yongfu Chen. Infrared radiation devices and typical applications. Beijing: electronic industry press, 2004.

[4] Shichang Jiang. An overview of the infrared measurement technology. Journal of measurement test, 1999(3):18 to 19 .

[5] Wenyan Tang. Sensors. Beijing: mechanical industry publishing house. (2006)

[6] Xihui Chen, Yinhong Zhang. LabVIEW programming from entry to the proficient in 8.20. Beijing: Tsinghua university press. (2007)

[7] Huoliang Liu, Sin Yang. STM32 library development practical guide. Beijing: mechanical industry publishing house. (2013)

[8] Xiangjun Hu. Circuit analysis. Beijing: higher education press. ( 2007)

[9] W.Richard Stevens (American). TCP/IP rounding, Volume 1: protocol. Interpreted by Jianhua Fan et. Beijing: mechanical industry publishing house. (2000) 